\title{
Treatment of Intractable Hemicrania Continua by Occipital Nerve Stimulation
}

\author{
${ }^{1}$ Sarah Miller MBBS, MRCP ${ }^{2}$ Laurence Watkins FRCS, PhD and ${ }^{1}$ Manjit Matharu FRCP, \\ $\mathrm{PhD}$ \\ ${ }^{1}$ Headache Group, Institute of Neurology and The National Hospital for Neurology and \\ Neurosurgery, Queen Square, London, UK \\ ${ }^{2}$ Department of Neurosurgery, Institute of Neurology and The National Hospital for \\ Neurology and Neurosurgery, Queen Square, London, UK
}

Prepared as letter for: JNNP

Title Character Count: 75

Word Count:999

Tables/Figures: 1

References: 5

\section{Corresponding Author:}

Dr MS Matharu

Senior Lecturer and Honorary Consultant Neurologist

Headache Group, Institute of Neurology and The National Hospital for Neurology and

Neurosurgery, Queen Square, London WC1N 3BG

Email: m.matharu@uclmail.net

Tel: $\quad+447595900535$

Fax: $\quad+447092120797$ 
Hemicrania continua (HC) is characterized by a strictly unilateral continuous headache with ipsilateral autonomic features and migrainous symptoms that is exquisitely sensitive to indomethacin ${ }^{1}$. As more than $30 \%$ of patients report side effects with indomethacin there is need for an effective, safe alternative. Although several drugs have been used in open-label studies, none offer the same magnitude of response.

Occipital nerve stimulation (ONS) has been performed in headache conditions such as chronic migraine and chronic cluster headache, both conditions with clinical similarities to HC, and appears to be a safe, potentially efficacious treatment even in those failing all available medical treatments. $^{2}$ We report the long-term follow-up of a new cohort of $16 \mathrm{HC}$ patients treated with ONS.

\section{PATIENTS AND METHODS}

Sixteen patients with a diagnosis of $\mathrm{HC}$ were implanted with bilateral ONS by a single specialist team between 2008-2013. Patients were diagnosed in accordance with the International Classification of Headache Disorders criteria ${ }^{3}$. All had unilateral headaches fully responsive to an oral or intramuscular Indotest ${ }^{4}$ but had suffered intolerable side effects or had contraindications to long term use of the drug. Patients had failed to respond to trials of at least five other medications with limited evidence of efficacy in HC: cyclo-oxygenase inhibitors, topiramate, verapamil, gabapentin, pregabalin, melatonin and flunarazine.

Procedures were offered under the supervision of local clinical effectiveness supervisory committee and ethical board approval for collection and publication of data obtained. Surgical procedure is described elsewhere ${ }^{5}$. 
Data on headache severity, frequency and intensity was collected at baseline and then every 36 months thereafter and was compared using paired t-tests.

\section{RESULTS}

The group consisted of nine males with a mean age of implant of 50.38 years (36-74) with median duration of HC 8.50 years (3-35). Patients had failed to respond to an average of 8.06 medications (5-12) and all had dose limiting side effects to indomethacin. Five patients had co-existent headache syndromes and kept separate diaries for these throughout follow-up.

Follow-up of the cohort was 38.50 months (11-86). The mean monthly moderate-severe headache days fell by $48.9 \%(\mathrm{p}=0.001)$ (Table 1$)$. A favorable response, defined as a more than $50 \%$ reduction in monthly moderate-severe headache days was observed in $50.0 \%(8 / 16)$ of patients.

The $50 \%$ response rate in those with multiple phenotypes was $60.0 \%(3 / 5)$ compared to $45.5 \%$ $(5 / 11)$ in the $\mathrm{HC}$ alone group. This difference was not statistically significant $(\mathrm{p}=1.000)$.

Adverse event profile was favorable with no infections, lead migrations or electrode erosions reported.

\section{DISCUSSION}

Current literature contains a total of 11 patients with ONS for $\mathrm{HC}$ with benefit suggested in at least five patients. However, there is diagnostic uncertainty in some of these patients due to lack of documented response to indomethacin. This new series is, therefore, the first to report on prospective ONS outcome data in a cohort of patients all meeting the diagnostic criteria for HC. 
Our open-label prospective series of 16 complex patients, including some with multiple phenotypes, adds support to ONS being a potentially efficacious treatment in intractable HC when indomethacin is not tolerated. A total of $50.0 \%$ of all patients showed a beneficial response with a reduction of $48.9 \%$ in monthly moderate-severe headache days. Daily indomethacin usage dropped by $65.1 \%$ in those continuing to take it.

It has been speculated that those with multiple phenotypes show less of a response to ONS than those with a single headache type. However, our data, all be it with small numbers, shows no difference between these groups. Given that ONS is a treatment reported as potentially useful for multiple headache conditions, our data suggests that those with multiple phenotypes should not be deprived of the treatment just because of concerns of poor outcome. However, a need exists for this to be examined over larger cohorts.

Improvements were observed in a number of outcome measures including moderate-severe headache days, pain intensity and daily headache hours. Given that there are no guidelines as to which measure is most appropriate for $\mathrm{HC}$ and that those recommended for chronic migraine are based on clinical trial measurements rather than "real-life" patients we feel that further work needs to be done to clinically validate outcome measurements in primary headache conditions. At present, the clinical improvements of a patient may not be being adequately captured by outcome measures, leading to a discrepancy between what is reported in the literature and what is seen in the clinic in ONS patients.

Occipital nerve stimulation has received major criticism over its adverse event profile. ${ }^{2}$ Previous cohorts of ONS in chronic migraine and cluster headache have reported high rates of infection, lead migration, erosion of electrodes through the skin and the need for repeated surgical interventions. ${ }^{2}$ However, our cohort shows a favorable adverse event profile even after prolonged follow-up. Only one patient required a surgical intervention (where the system 
was changed to one suitable for a rechargeable battery) and no infections or lead migrations. Our data supports the concept that adverse events can be minimized by ensuring implants are carried out by a small number of highly experienced centers.

There are limitations to this data, including those inherent to any unblended series, but given the rarity of $\mathrm{HC}$ it is unlikely that an adequately powered, randomized trial with adequate placebo will be carried out anytime in the near future. However, strengths of the series include the use of prospective data and a prolonged follow-up period. The cohort was also based on clinical need and as such will be transferable to other patients in similar specialist headache centers. Although a reduction in moderate-severe headache days of $48.9 \%$ may not appear positive to some, such a response in a highly intractable group of chronic pain patients should, instead, be viewed with cautious optimism.

In conclusion, there is a need for alternatives to indomethacin in $\mathrm{HC}$ due to intolerable side effects and our series suggests that ONS may provide a useful and safe treatment option even after prolonged follow-up. However, ONS should be reserved for those failing to respond to adequate numbers of other medical treatments.

1. $\quad$ Bordini C, Antonaci F, Stovner LJ, Schrader H and Sjaastad O. "Hemicrania continua": a clinical review. Headache. 1991; 31: 20-6.

2. Magis D and Schoenen J. Advances and challenges in neurostimulation for headaches. Lancet Neurol. 2012; 11: 708-19.

3. The International Classification of Headache Disorders, 3rd edition (beta version). Cephalalgia. 2013; 33.

4. Antonaci F, Costa A, Ghirmai S, Sances G, Sjaastad O and Nappi G. Parenteral indomethacin (the INDOTEST) in cluster headache. Cephalalgia. 2003; 23: 193-6.

5. Miller S, Watkins L and Matharu M. Long-term outcomes of occipital nerve stimulation for chronic migraine: a cohort of 53 patients. J Headache Pain. 2016; 17: 68. 